\title{
A Review Research of Errors Caused in Auditing Process
}

\author{
Shiliang Xia \\ Department of Economics and Management, Huaiyin Teachers College, Huaian 223300, China \\ E-mail: xiashiliang2006@126.com
}

\begin{abstract}
There is a significant growth of behavior economics in the last decade. The goal of the behavior research is aimed at the deviations from rationality in order to improve the predictive power of economic models. In this paper, attached to the specific auditing process, I want to classify the neglecting relevant information due to judgment by representative which causes the bias, or the error of auditing results.
\end{abstract}

Keywords: Auditing Process, Auditors, Information Neglect

\section{Background}

As we know that bias research, or the error research in auditing has been conducted with both psychology -and economics-based experiments. While both approaches examine similar phenomena, they stand rather separately apart from one another. Recommendations for their synthesis have regularly been made in the past, the number of co-citations between them remained very low. Over the time the focus of psychology -based research has shifted. The first wave of research replicated the experiments of Tversky and Kahneman (1974) examining the anchoring bias and the representative ness heuristic in the audit context. The second wave developed with the introduction of the belief-adjustment model by Hogarth and Einhorn (1992). The model allowed for a more precise testing of sequential decision making which made it easier to examine order effects or confirmatory processes. In a third wave beginning at the end of the 1990s, bias research became more and more issued oriented. Biases that affected auditor liability, like the hindsight bias, and heuristics that affected auditor independence, like the acceptability heuristic were investigated.

\section{Definition}

During the auditing process there exist some errors caused by the neglecting relevant information due to judgment by repetitiveness which means that the decision-maker focus on highly representative and salient factors, while neglecting other potential more important considerations. For the evaluation of random drawn samples of data, this means that people focus too much on the distribution indicated by the sample, while neglecting the size of the sample. Similarly people tend to believe that very small sample sizes are highly representative of the underlying population integrated this finding into an economic model.

\section{Categorization}

\subsection{Sample size neglect}

As auditors very often use non-random methods for sampling and non-methods for evaluating these samples, the danger might exist that auditors overestimate the power of small samples due to the representative ness heuristic. While a study by Kachelmeier and Messier(1990)found that auditors are prone to this error, and that they choose to draw too small sizes when compared to size demand by audit standards, a later study did not find this effect as a new version of the audit standards required smaller sample sizes.

\subsection{Base rate neglect}

Decision-makers often tend to focus only on the direct information context of evidence, without considering it in light of prior knowledge of base rates. Explanations for this are that base rates are not perceived to be salient features of the decision, and that people have difficulties to understand the concepts of base rates. However, depending on the context, base rate are not always neglected. Auditors seems to be no exceptions, they also neglect base rates in auditing tasks. Joyce and Biddles (1981) argued that base rates are less often neglected for similar tasks, but Holt (1987) showed that the apparent decrease in the neglect of base rates is not due to experience effects, but to the wording used in the task description. The findings of Tuttle (1996) suggest that experience might improve the choice of the relevant sub-sample, which would improve the decision quality of auditors even when they are prone to the base rate neglect. The context dependency of the base rate neglect was also demonstrated by Kida (1984), who showed that auditors use more often base rates when they are presented in a way that highlights the causal link between the base rate and the relevant population. 


\subsection{Source credibility neglect}

In judgment solely based on the representative ness of the message, the inherent credibility of the source would be neglected. While Joyce and Biddle(1981) found that auditors neglect the credibility of the source, later research came to the conclusion that auditors consider various forms of source reliability in their judgment, for example ,source competence, and source integrity. Auditors consider source reliability not only in their judgment, but also directed their information search towards more reliable sources. As in Bamber(1983), auditors sometimes even excessively discounted evidence from unreliable sources.

Auditors showed a slight focus on negative evidence. For example, changes in source reliability are given special consideration when that change was negative, or when the source content is associated with negative consequences. The general finding that auditors consider source reliability is also corroborated by research examining the effects of the internal control strength on audit planning. One reason might be that source credibility is a salient feature in auditing.

\subsection{Conjuction fallacy}

Basic statistical theory tells us that probability of a certain event X must be higher than the probability that X happens in conjunction with another event $Y$. However when the combination of two conjunctive events is highly representative for a certain scenario, people tend to estimate the likelihood of this combination higher than the likelihood of both single event. Equivalently, the likelihood of a single representative event is judged to be higher than a disjunctive combination of this event with a less representative event. The conjunction fallacy is moderated when probabilities are presented in the form of frequencies.

In auditing an early study by Joyce and Biddle (1981) indicates that the experience of auditors eliminates the conjunction fallacy. However, this result was probably driven by a lack representative ness in the conjunction event described. Further study showed that experience might even enhance the conjunction fallacy as it goes along with a better sense of the representative ness of events. For example, auditors were prone to the conjunction fallacy when estimating the likelihood of possible error combinations that were highly representative due to the principle of double bookkeeping. Similarly, they overestimate the likelihood of combinations of plausible error causes for extreme changes in a key financial indicator, especially when one of the plausible errors triggered audit implication. Requiring auditors to state the likelihood state the likelihoods of the conjunctive event as well as the likelihoods of the single events helps them to reduce the bias in their judgment due to the conjunction fallacy.

\section{References}

Tversky and Kahneman. (1974). Belief in the Law of Small Numbers. Psychological Bulletin 76(2):105-110.

Hogarth and Einhorn. (1992). The Belief Adjustment Model. Cognitive Psychology 24(1):1-55.

Kachelmeier and Messier. (1990). An Investigation of the Influence a non-statistical Decision Aid on Auditors Sample Size, Accounting Review (1):209-226.

Joyce and Biddles. (1981). Anchoring and Adjustment in Probabilistic Inference in Auditing, Journal of Accounting Research 19(1):120-145.

Tuttle. (1996). Using Base Rate Frequency Perceptions to Diagnose Financial Statement Error Causes, Auditing 15(1):104-121.

Kida. (1984). The Effect of Casualty and Specificity on Data Use, Journal of Accounting Research22 (1):145-152.

Bamber. (1983). Expert Judgment in the Audit Team: a Source Reliability Approach, Journal of Accounting Research21 (2):396-412. 